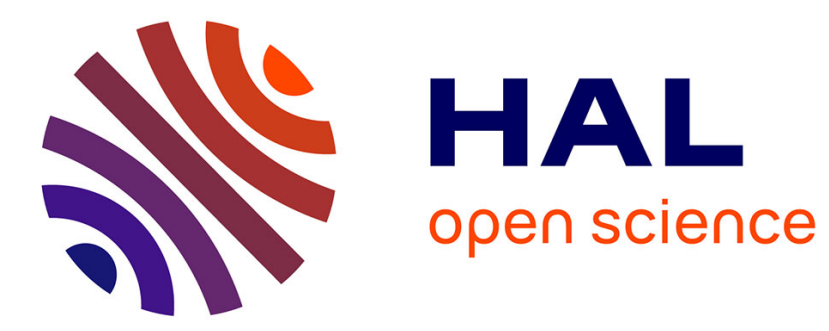

\title{
Le système des lieux dans le Journal : le cas des descriptions d'intérieur
}

\author{
Dominique Pety
}

\section{To cite this version:}

Dominique Pety. Le système des lieux dans le Journal: le cas des descriptions d'intérieur. Cahiers Edmond et Jules de Goncourt, 2003, Les cent ans du premier prix Goncourt, 1 (10), pp.233-267. 10.3406/cejdg.2003.921 . hal-02468862

\section{HAL Id: hal-02468862 \\ https://hal.univ-smb.fr/hal-02468862}

Submitted on 6 Feb 2020

HAL is a multi-disciplinary open access archive for the deposit and dissemination of scientific research documents, whether they are published or not. The documents may come from teaching and research institutions in France or abroad, or from public or private research centers.
L'archive ouverte pluridisciplinaire $\mathbf{H A L}$, est destinée au dépôt et à la diffusion de documents scientifiques de niveau recherche, publiés ou non, émanant des établissements d'enseignement et de recherche français ou étrangers, des laboratoires publics ou privés.

\section{(ㅇ)(1) $\$$}

Distributed under a Creative Commons Attribution - NonCommercial - NoDerivatives $\mid 4.0$ 
Le système des lieux dans le Journal : le cas des descriptions d'intérieur

Dominique Pety

\section{Citer ce document / Cite this document :}

Pety Dominique. Le système des lieux dans le Journal : le cas des descriptions d'intérieur. In: Cahiers Edmond et Jules de Goncourt n¹0, 2003. Les cent ans du premier prix Goncourt. pp. 233-267;

doi : https://doi.org/10.3406/cejdg.2003.921

https://www.persee.fr/doc/cejdg_1243-8170_2003_num_1_10_921

Fichier pdf généré le 17/05/2018 


\section{Le système des lieux dans le Journal : le cas des descriptions d'intérieurs}

Tous les thèmes abordés dans le Journal suscitent l'évocation des espaces qui leur sont associés : les restaurants, théâtres et bals fréquentés appellent des commentaires sur la décoration des salles; les milieux de la presse transparaissent notamment dans les bureaux des principaux journaux, les domiciles des différents journalistes; la maladie éprouvée ou étudiée pousse à évoquer l'hôpital, à décrire le salon de tel grand chirurgien ... Et les lieux ne sont pas seulement nommés ou suggérés, mais visités, décrits, jugés, à destination parfois de l'œuvre romanesque. Ils constituent ainsi une donnée sousjacente à toutes les problématiques, et dessinent, semble-t-il, un système de représentation spécifique à l'intérieur du Journal. C'est pourquoi il serait intéressant de dresser en quelque sorte une cartographie du Journal, qui permettrait de distinguer les espaces - sociaux (les lieux où l'on vit), culturels (les lieux où l'on pense), esthétiques (destinés d'emblée à l'art, comme les musées et salons, ou investis ponctuellement par un regard d'esthète : les paysages, ciels et jardins...) - et ces espaces à l'opposé qui se transforment en nonlieux : la province (celle où l'esprit s'étiole et où le collectionneur ne trouve pas d'objet d'art), l'étranger (outre les pays découverts par le voyage, il y a ceux où il est inutile d'aller parce qu'il suffit de les imaginer - le Japon - ou de s'en tenir aux on-dit: les ÉtatsUnis...).

La tâche est vaste, et on s'en tiendra ici aux seuls espaces intérieurs, en effectuant une série d'allers-retours avec d'autres textes des Goncourt qui comprennent aussi des descriptions de ce genre et aident à préciser les motivations multiples de leur présence dans le 
Journal, tant il est vrai que ce dernier s'inscrit à la croisée de plusieurs genres. On s'efforcera, en outre, de suivre au plus près la perspective chronologique, par laquelle on devient, au cour du Journal, sensible à la maturation et à l'évolution de ses divers projets d'écriture.

Mais il faut peut-être d'abord préciser ce qu'on entend ici par "lieux", et proposer quelques éléments pour une typologie plus générale des (mentions de) lieux. Deux aspects sont semble-t-il à prendre en compte : les données référentielles d'une part ; la modalité de leur insertion dans le discours du Journal d'autre part. Or, c'est plutôt ce second aspect qui conditionne en fait le premier; on peut ainsi classer les lieux :

1. En fonction de l'étendue de leur mention. Le lieu peut être nommé (" chez la princesse ": le but est alors seulement d'indiquer le contexte, l'origine d'une parole entendue, ce n'est pas l'aspect physique du lieu qui intéresse), ou non (il faut alors le déduire à partir d'un détail, d'un personnage). Entre la mention seulement nominale et la description minutieuse du décor, on devine la variété des indications de lieux.

2. En fonction du narrateur. On peut distinguer un premier degré et un second degré, selon que les Goncourt rapportent ce qu'ils ont vu eux-mêmes ou le propos d'un autre personnage qui a été témoin: les mentions ne relèvent pas alors de leur observation, mais de leur choix'.

3. En fonction de l'identité géographique ou sociale du lieu. Scènes d'intérieur (bureaux de L'Éclair, maison de Janin, boutique du marchand de tableaux Peyrelongue...) ; scènes d'extérieur (rue, boulevard...) ; lieux mixtes, clos mais en mouvement, et d'où l'on regarde surtout dehors (le train). Cas particulier des "effets de ciel ", sans ancrage géographique précis, assez proches de l'œuvre d'art, plus décontextualisés encore, pure gratuité et pur anonymat (non signés, si ce n'est par le descripteur).

1. Il faudrait se demander quels sont les critères de ce choix, et s'il arrive qu'ils soient explicitement formulés. Le problème de la sélection, manifeste pour la publication, se pose aussi pour la consignation. 
4. En fonction du statut réel ou fictif du lieu; et parmi les lieux fictifs, il faudrait aussi distinguer lieux imaginés à destination de la fiction littéraire, lieux désirés, lieux rêvés (un certain nombre de rêves sont transcrits dans le Journal, et parmi eux des rêves centrés sur le lieu, des rêves de lieux).

On fera ici le choix de s'en tenir aux lieux non seulement nommés, mais décrits (même lorsqu'ils le sont brièvement) ${ }^{2}$, et décrits au premier degré ; mais on tiendra compte tout à la fois des lieux réels et des lieux fictifs (littéraires et rêvés). En outre, une restriction thématique s'impose pour la cohérence du propos : on s'intéressera aux intérieurs (en excluant ainsi les évocations de paysages qu'il faudrait étudier à part, en interaction avec le discours sur la peinture) ; ces intérieurs pourront renvoyer à des lieux publics, institutionnalisés (le tribunal, l'hôpital...), ou aux lieux d'habitation, aux espaces intimes.

On peut d'emblée préciser la part restreinte des lieux dans les débuts du Journal, marqués par une alternance de brèves remarques $^{3}$ et de morceaux plus longs ${ }^{4}$. La forme du Journal se cherche entre la note de deux lignes et l'excursus descriptif plus détaillé, le Journal hésite entre le recueil de pensées (La Rochefoucault, Chamfort) et la galerie de portraits (La Bruyère). Mais c'est surtout l'auditif qui prime sur le visuel : le Journal rend davantage compte des paroles que des comportements. Les notations spatiales, les éléments de décors apparaissent d'abord à l'appui des portraits'.

2. Sinon il faudrait procéder avec beaucoup plus de détails, année par année, avec des synthèses par période, et dans une perspective essentiellement sociologique: dans les années 1850 , les Goncourt fréquentent surtout les ateliers des paysagistes, les bureaux des petits journaux, les petits théâtres; dans les années 1860, le bureau de la Gazette des Beaux-Arts et le salon de la princesse Mathilde, etc.

3. "La sculpture anglaise et les romances de Loïsa Puget sont sœurs"; "Ah! si l'on avait un secrétaire de ses ivresses ! "; "À Paris, un pauvre sur treize individus " (1852, I, 35-36). Les références au Journal sont données dans l'édition Laffont (Bouquins, 1989, $3 \mathrm{vol}$.), avec l'indication du volume et de la page après la date.

4. "J'ai eu deux cousins [...] " ou " Portraits en omnibus ", I, 38-40.

5. Timoléon de Villedeuil va à la messe, est accueilli par ses domestiques sur le perron lorsqu'il regagne son château, il a son "prie-Dieu en tapisserie dans sa chambre " et des "meubles de la Restauration, des fauteuils en soie"; Léonidas Labille couche dans une chambre sans rideaux et sans table de nuit (1852 I, 39-40). Si les notations spatiales 
La part des lieux dans le projet du Journal - tel qu'il est formulé et théorisé a posteriori, par exemple dans la préface de 1872 - peut aussi sembler réduite. La référence au genre des mémoires et le primat de l'observation pourraient conduire à octroyer aux données matérielles une place de premier plan. Mais cette préface insiste davantage sur les personnes, leurs paroles, leurs gestes, et ne dit rien des lieux, des décors, des demeures, dont on s'attend alors à ce qu'ils soient secondaires dans cette galerie de portraits, et présents seulement dans la mesure où ils contribueront à la caractérisation des personnes.

On peut se demander ce qu'il en est véritablement, et si cette évocation des lieux n'acquiert pas au fil du Journal une certaine autonomie, jusqu'à jouer, après 1870 surtout, un rôle de premier plan.

\section{L'apparition des descriptions de lieux dans le Journal Le modèle des notes de voyage}

Les premières indications de décor dans le Journal prennent deux formes distinctes. Soit il s'agit d'une présentation circonscrite et intégrée dans un récit : on dépeint brièvement le décor d'une action qui occupe quant à elle le premier plan. Cette mise en récit est sensible dans l'effort de distanciation temporelle, dans l'écriture au passé simple et à l'imparfait, de faits qui sont en réalité tout à fait récents :

Nous soupons beaucoup toute cette année [...].

L'autre jour, ou plutôt l'autre nuit - il était quatre heures du matin. C'était dans le cabinet $\mathrm{n}^{\circ} 7$ de la Maison d'Or, entre les murs à panneaux entourés de baguettes dorées, avec de grosses

manquent à cette époque, il en est de même pour les notations temporelles : l'année 1851 est réduite au mois de décembre et ne comporte qu'une date, celle du 21 décembre 1851 , mais cette journée - mouvementée - est quant à elle détaillée, de même que les lieux visités ce jour-là (" au cinquième, rue Mogador, dans l'appartement de l'actrice qui rapporta Musset de Russie. Une vierge byzantine [...] une glace d'actrice, qui s'ouvre à deux battants, un miroir triptyque [...] " 1851, I 31). Pour 1852, trois indications de date seulement. 
fleurs rouges et blanches, éclatantes et de larges feuilles imitant par leur relief le laque de Coromandel. Sur le canapé de velours rouge, une femme rousse était couchée $[\ldots]^{6}$.

Soit, au contraire, les indications de décor ne sont pas subordonnées à un récit et apparaissent sous forme de notations juxtaposées (brèves phrases nominales ou au présent, exclusivement référentielles, dépourvues de jugement de valeur) : c'est le modèle des notes de voyage prises par les deux frères lorsqu'ils traversèrent la France jusqu'en Algérie, de juillet à décembre 1849, comme prélude à une carrière de peintres ${ }^{7}$.

On a un exemple frappant de ces deux manières d'écrire, caractéristiques des débuts du Journal (distanciation temporelle de la mise en récit, ou présent des notations descriptives juxtaposées), lorsque les Goncourt évoquent, en 1854, la maison d'un certain Turcas à Sainte-Adresse ${ }^{8}$. Après quelques phrases au présent qui précisent les données événementielles ("Fin août. Nous allons passer un mois aux bains de mer, auprès du Havre, à Sainte-Adresse "), se déploient deux textes distincts. D'une part, le récit des amours de Jules avec une actrice, d'autre part, des notations en style télégraphique ("Sur un dressoir rustique, faïence bleue [...]. Salle à manger : plafond en perse tendue, plissant vers le centre; au mur, trois lithographies [...]"), précédées de "Sainte-Adresse: maison de Turcas ", qui est comme l'indication de lieu laissée par l'artiste en haut de son croquis. Et le fait que l'on soit en province, et que la chose soit rappelée en tête de ces indications descriptives, montre qu'on est bien dans le registre de la note pittoresque du récit de voyage.

6. Journal, novembre 1852 , I, 59. Voir un semblable dispositif en janvier $1853(1,61)$ : "La curiosité de ces bureaux était le bureau de Villedeuil [...]".

7. Le manuscrit manque, mais des lettres à des proches nous ont conservé des extraits Voir J. de Goncourt, Lettres, Charpentier, 1885, et E. et J. de Goncourt, Lettres de jeunesse inédites, éd. Alain Nicolas, PUF, 1981.

8. Journal, I, 104 sq. 


\section{L'influence de Gavarni}

Le dessinateur Gavarni, que les deux frères rencontrent très tôt au début de leur carrière d'écrivains, a dû jouer un rôle essentiel dans le goût qu'ils acquièrent peu à peu des indications de décors, et particulièrement des descriptions d'intérieurs.

Le Journal conserve la trace des nombreuses conversations avec l'artiste, regroupées, par Edmond, sous le titre de "Gavarniana ". On découvre que ces conversations ont porté notamment sur les lieux habités par Gavarni, synthétisés en 1855 sous une rubrique "Habitations" qui ne comporte en fait que trois mentions d'adresses, mais qui consigne probablement une évocation orale plus détaillée ${ }^{9}$. Ce que suggère une remarque similaire, en 1856 : "Nous parle de l'intérieur des gens, de l'intérieur de Daumier, qu'il a vu ainsi : une grande pièce, autour d'un poêle en fonte chauffé à blanc; des hommes assis par terre, une bouteille de vin chacun [...] dans un coin, une table avec tout le désordre des choses lithographiques entassées " 10 . Et le Journal enregistre aussi le goût de Gavarni pour la transformation, l'aménagement, la décoration: "parlant d'arrangements de sa maison d'Auteuil, bronzes, treille dans la salle à manger, etc. $"{ }^{11}$.

On trouve confirmation des propos que les Goncourt rapportent dans des textes de Gavarni lui-même, des carnets qu'il s'est plu à tenir tout au long de sa vie, et dont les Goncourt s'inspirent dans la monographie qu'ils rédigent à la fin des années $1860^{12}$. Gavarni a pris soin de décrire les lieux qu'il a occupés, cet appartement impasse des Minimettes à Bordeaux ("Voici la description de cet intérieur dans une lettre écrite par le jeune Chevalier à sa mère, en janvier $\left.1825[\ldots] \|^{13}\right)$, cette autre maison qu'il occupe ensuite dans la même ville ("Une étrange maison, cette maison Marcadée, et dont la description, longuement détaillée, pleine de couleur et d'effet,

9. Journal, I, 123.

10. Journal, 25 décembre 1856, I, 226.

11. Journal, septembre $1854, \mathrm{I}, 110$.

12. Gavarni, l'homme et l'cuvre (1873), Fasquelle, 1925.

13. Ibid., chap. V, p. 7. 
semble, sous la plume de l'écrivain de vingt-et-un ans, un tableau feuillu de vieille maison provinciale, pareil à une copieuse page de Balzac, écrite dix ans avant lui "; "De sa chambre [...] l'artiste nous a laissé l'inventaire [...] $\left.{ }^{14}\right)$. Et les Goncourt soulignent plus généralement "l'attachement que le peintre semble avoir pris à toutes les époques de sa vie pour les endroits qu'il habitait, l'affection qui lui venait pour des murs d'habitude et qui, à presque chaque changement de demeure, lui en faisait crayonner la mémoire $[. .] "$.15 .

Cette préoccupation de Gavarni pour les lieux qu'il a habités trouve sa traduction dans ses dessins, au décor toujours précis et évocateur : "La borne, le trottoir, le mur, le lambris, la chaise, les rideaux parlent au second plan des lithographies de Gavarni, aussi éloquemment que parlent, au premier, l'habit de l'homme et la robe de la femme. Ils sont aussi significatifs, aussi dénonciateurs; un détail, un rien, un coin entrevu derrière une scène, en complète le sens et en accentue la réalité " ${ }^{16}$. Dès 1859 d'ailleurs, les Goncourt avaient vu en Gavarni un peintre de mœurs, pour ces " modes des choses" dont il est le témoin fidèle (" les fonds mystérieux des appartements, le clair-obscur des alcôves, les portières de soie tombantes et serrées à la taille, les potiches ventrues, les cadres lourds, les tapis sourds ${ }^{17}$ ). Sa démarche est ainsi celle d'un historien consignant les données du quotidien ("grands historiens sans le dire qui s'appellent Abraham Bosse ou Longhi, Hogarth ou SaintAubin, Goya ou Gavarni ! "). L'histoire selon les Goncourt sera elle aussi une histoire sociale, une histoire des mours, et appellera de même la description du cadre de vie.

14. Ibid., chap. VII, p. 10.

15. Ibid., chap. XXV, p. 40 ("l'atelier de la rue Saint-Lazare à Paris"). Voir aussi le Journal décrivant, fin mai 1860 (I 565), "un très curieux dessin représentant la chambre de Gavarni à Tarbes ", description reprise en note dans Gavarni, chap. X, p. 17.

16. Ibid., chap. LXXXIII, pp. 145-146 ("Le décor de ce monde, le fond mobile et changeant sur lequel se détache toute cette humanité, le chez soi et la rue n'ont pas été négligés par Gavarni. Il a voulu que son peuple parisien fût représenté dans les milieux exacts et précis où se passe et s'agite sa vie [...]").

17. Gavarni, D'après nature, 4e dizain par Edmond et Jules de Goncourt ", 1858 (texte reproduit dans Gavarni, CEures choisies, avant-propos de Paul-André Lemoisne, Horizon de France, [s.d.]). 
On peut aussi mesurer l'influence de Gavarni dans le choix des descriptions de décors quand on constate que la demeure d'Auteuil, où les Goncourt ont rendu visite à l'artiste, figure parmi les toutes premières évocations d'intérieurs du Journal. En outre, au fil des pages, on peut suivre les progrès de la démarche descriptive des deux frères. La première visite, en 1852, ne donne lieu qu'à quelques remarques en style paratactique : "Petit lit de fer étroit de moine. Deux corps de bibliothèque. Un couteau dans un livre ayant pour titre Le Cartésianisme " ${ }^{18}$. En 1855, mêmes notations juxtaposées, à peine plus précises, mais qui concernent d'autres parties de la maison : "Intérieur en haut, dans un petit atelier mansardé. Un poêle en fonte. Tout autour, un mur, des plans imprimés ou décalqués sur papier végétal, du Palais-Royal et de la rue de Rivoli auprès du Palais-Royal. Un grand bureau avec des livres; spécialement le livre relié en vélin, où il écrit. Près de la fenêtre, son chevalet " ${ }^{19}$. Le 17 mars 1858, on a droit à un inventaire beaucoup plus détaillé de l'atelier mansardé $e^{20}$ :

À Auteuil, un atelier mansardé, une seule fenêtre, une cheminée de marbre noir, une console en acajou et en bronze. Sur la pendule, le buste en plâtre, singulièrement fin, du fils de $\mathrm{M}$. Magne.

Contre le mur, un porte-lorgnette, un thermomètre en bois doré, restes de son ancien mobilier. Des deux côtés de la porte, deux bibliothèques en acajou, surmontées d'étagères : livres modernes, livres de mathématiques, livres d'amis. Contre le mur, une palette chargée de tons de gouache [...].

En outre, le style paratactique cède ici la place à la mise en scène de l'artiste au travail : "Auprès de la fenêtre, auprès d'un grand panier

18. Journal, I, 49-50. Comme l'indique la note de R. Ricatte, l'apparence extérieure de la maison, le parcours rapide de l'habitation dans son ensemble, l'atelier dans le jardin, sont en fait un emprunt au Gavarni de 1873 pour la version publiée du Journal. La juxtaposition des deux passages, celui de 1852 et celui de 1873 , rend plus frappante la différence de styles : les brèves notations juxtaposées tranchent avec la description narrativisée, ajoutée ensuite.

19. Journal, I, 119.

20. Journal, I, 335-336. Il sera réécrit dans le Gavarni de 1873 : " Une seule fenêtre éclairait la pièce. Une cheminée en marbre noir, sur laquelle était une pendule dans une boîte en acajou, restait l'hiver sans feu : un poêle de fonte chauffait la pièce ". 
de tapisserie, brodé par sa femme, donné à sa mère et gardé comme souvenir de sa mère, dans un grand fauteuil d'acajou à velours vert, les pieds dans une chancelière, il travaille sur un chevalet, une espèce de petit guéridon à côté de lui ». Dernière évocation du lieu du vivant de l'artiste le 22 mai $1859^{21}$. Assez rapide, elle concerne le bas de la maison, et pour la première fois, on constate la disparition totale de la parataxe.

\section{L'influence de la collection}

J'ai montré ailleurs ${ }^{22}$ comment la réorganisation de la collection de dessins des frères Goncourt, dans les années 1857-1858 (revente sélective, nouveau carnet consignant plus méthodiquement les achats, projet de préface pour un catalogue) allait de pair avec l'apparition dans le Journal de mentions de plus en plus fréquentes des acquisitions, lesquelles amènent bientôt des évocations du cadre de vie, où les objets acquis figurent $\mathrm{t}^{23}$.

C'est aussi en 1858 qu'apparaissent les premières descriptions minutieuses et quasi exhaustives d'intérieurs de parents ou d'amis : la chambre de Blanche Passy, le cabinet de Léonidas Labille ${ }^{24}$, deux lieux privés très différents, que rapproche l'appartenance de leur propriétaire respectif à la sphère intime des scripteurs. A partir du moment où les Goncourt prennent conscience de l'intérêt, de la légitimité de l'évocation de leur propre intérieur dans le Journal, la démarche va se généraliser progressivement, s'étendant d'abord aux proches. La description d'intérieur conquiert ainsi droit de cité

21. Journal, I, 459.

22. Les Goncourt et la collection. De l'objet d'art à l'art d'ecrire, Droz, 2003, p. 31 sq.

23. Il faudrait essayer de périodiser finement le Journal en fonction de problématiques diverses, de façon à faire apparaître des charnières importantes, dans une perspective interne à l'œuvre (afin de voir comment le projet du Journal se transforme, comment il enregistre les évolutions dans l'esthétique plus générale des deux frères), dans une perspective externe (pour voir comment le Journal reflète l'évolution sociale des deux frères, et les évolutions culturelles du XIX siècle en général). Le Journal des Goncourt est une des façons dont le XIX siècle se pense, et cette périodisation pourrait rejaillir sur notre façon d'appréhender la $2^{\mathrm{c}}$ moitié du siècle au sens large.

24. Journal, 5 septembre 1858, I $397 ; 26$ septembre 1858, I, 402. 
dans le texte, parce qu'elle dévoile l'intime, au même titre que l'apparence physique ou le portrait moral de la personne : on mesure la distance parcourue entre le portrait de Léonidas Labille en 1852 et celui qui commence par cette longue description d'un lieu, le 26 septembre 1858 : "Le cabinet de mon cousin est ainsi $[\ldots] \|^{25}$.

L'étude de ces premières tentatives descriptives montre bien à la fois les divers modèles dont s'inspirent les Goncourt, et l'évolution d'une forme - descriptive - et d'un motif - le cadre physique, et particulièrement, le décor intérieur ${ }^{26}$ - qui cherchent à définir leur place et leur statut dans un texte, le Journal, à l'identité elle-même problématique. Le dispositif repéré au début des années 1850 va se confirmer à plus large échelle. Soit la description est insérée dans un autre texte, narratif ou dramaturgique, et elle est maintenue dans un rôle subalterne, en dépit de la précision qu'elle peut atteindre : le salon de Gisette Dennery sert de décor (" je suis assis au coin de la cheminée, le coude à une table où sont rangées une armée de brosses montées en ivoire, dans une pièce chaude, fermée, douillette, à grands ramages de perse [...] ") ou de prétexte (dans le salon, la collection de monstres orientaux) au marivaudage de Jules avec la jeune femme ${ }^{27}$. Soit la description, autonomisée, cherche, dans des directions diverses, sa légitimité. Dans les notes de voyages, les carnets du dessinateur Gavarni, elle était un support à l'activité artistique; en rendant compte de leur propre intérieur de collectionneurs, les Goncourt découvrent au lieu lui-même, et au texte qui en rend compte, une motivation esthétique. C'est aussi la proximité du lieu avec la personne (à commencer par la leur), et le critère de l'intime qui peuvent légitimer la description du cadre, en

25. Journal, I, 40 et I, 402-404.

26. C'est un type de description spécifique, qu'il faudrait confronter à d'autres, comme les descriptions de paysages, qui se déploient elles-mêmes progressivement dans le Journal, et qui semblent partager une origine commune dans les notes de voyage des apprentis peintres.

27. Il conclut de fait, au moment de sortir : "Ma parole d'honneur, si nous n'avons pas l'air de jouer un proverbe ! je fais des fausses sorties comme dans Musset, n'est-ce pas ?" (Journal, 29 décembre 1859, I, 506-511). 
la rendant complémentaire du portrait. Valeur documentaire du lieu comme enveloppe de l'intime, qui ira croissant quand le lieu se fera en outre cadre de l'écriture; dès lors, c'est la perspective esthétique qui s'imposera de nouveau, et qui expliquera la prégnance du motif de la maison dans les années 1870-1890. Avant d'en venir là, il faut analyser plus en détail la place que lui réserve le Journal, selon qu'il fonctionne comme réservoir pour l'œuvre romanesque, recueil de documents pour l'histoire du présent, ou machine de gucrre pour forger et imposer l'image de l'homme de lettres.

\section{La question de la description romanesque La théorie de l'observation}

Le Journal va devenir au cours des années 1860 recueil de notes préparatoires pour l'écrivain qui fonde sur l'observation sa pratique romanesque : les choses vues seront consignées à destination de l'écriture et non plus de la peinture, comme c'était le cas des " notes de voyage». Ce qui implique des modifications formelles (on n'enregistre peut-être pas de la même manière ce qui doit servir à l'une ou à l'autre), mais d'abord de nouveaux critères de sélection : ce n'est plus désormais le pittoresque qui est privilégié.

Celui-ci semble encore prévaloir dans les années 1850 , lorsque les deux frères décrivent la rue et ses boutiques, dans un style paratactique, sans jugement de valeur, et avec des détails très précis : "Sur une planche, une burette en fer blanc à huile, une assiette, sur laquelle il y a une vieille croûte de pain et une fourchette et un couteau sales : plus loin, un bol en faïence; plus loin, trois à quatre assiettes les unes sur les autres ${ }^{28}$. C'est là une nature morte, qui évoque irrésistiblement la peinture de Chardin. La perspective esthétique domine aussi dans l'évocation d'une maison de filles, constituée surtout d'indications de lumière et de couleurs, en une liste de détails juxtaposés ${ }^{2}$.

28. Journal, novembre 1855, I, 165. Voir aussi juillet 1855, I, 139.

29. Journal, " un samedi de juillet 1856 », I, 191. 
Peu à peu, néanmoins, l'intérêt documentaire commence à motiver les descriptions de lieux, particulièrement lorsque ceux-ci sont atypiques, tel cet appartement de photographe : l'énumération ne porte plus sur des détails visuels; elle déploie un vocabulaire plus technique, mieux adapté à la dénomination d'un lieu nouveau ("Labyrinthe à deux étages de salons, cabinets, recoins, ateliers, officines, séchoirs, greniers à produits chimiques - tout l'attirail photographique. Encombrement de daguerréotypes, stéréoscopes, photographies... $\left.{ }^{30}\right)$. Elle se double en outre d'une généralisation, et d'une prise de parti : "Il y a comme une mort dans cet embaumement de la ressemblance; un funèbre portrait de la vie, toutes ces faces diverses amoncelées et rangées dans des boîtes comme dans une bière, toutes ces chairs et ces yeux morts, sans couleur, ni physionomie ".

On voit donc apparaitre un nouveau type de motivation descriptive, qui pousse à consigner des éléments de décor pour leur valeur documentaire. Mais l'évocation reste souvent succincte. Un tribunal de province, vu le 26 juin 1860, donne surtout l'occasion, après une brève esquisse du cadre ${ }^{31}$, de camper les acteurs du procès et de transcrire les discours dans leur détail. C'est ce contenu humain qui suscite le désir d'écriture (" Nous songeons à la jolie chose ironique qu'il y aurait à faire avec un tribunal comme celui-ci et un président pareil, avec ses petites prosopopées et sa morale prudhommesque »). On peut faire le même constat à la fin de la décennie : les deux frères ont pourtant en tête un projet précis (ils travaillent à ce qui sera La Fille Élisa), ils sont à Paris dans un lieu important, luxueux, "la nouvelle salle de la cour d'assises", qu'ils ne connaissent pas et qui pourrait appeler une évocation détaillée. Mais après une mention liminaire ${ }^{32}$, la priorité est donnée au déroulement des audiences.

30. Journal, 21 décembre 1856, I, 224.

31. Journal, I, 577 ("Une salle blanchie à la chaux, où passe le tuyau d'un poêle, des fenêtres à jalousies. Un Christ sur un mur, qui regarde un Napoléon de plâtre ").

32. Journal, 10 mars 1869, II, 207 ( Nous sommes dans la nouvelle salle de la cour d'assises. Les dorures, des tableaux, un plafond reluisant, partout du confortable et du luxe joyeux et criard, les heures de l'anxiété qui sont là sonnées par une pendule d'or "). 
Donc, soit le lieu est rapidement décrit pour donner priorité à l'humain, soit les Goncourt s'y attardent parce qu'ils y découvrent en outre cette valeur esthétique qui motivait leurs premières évocations de décors. On peut citer le cas de la prison pour femmes de Clermont de l'Oise, qu'ils visitent lors d'un séjour chez leurs cousins Lefebvre de Béhaine. Lorsqu'ils s'arrêtent sur la salle de travail des prisonnières, on comprend rapidement pourquoi :

Cela était clair, transparent, d'un bleu blanc et froid. Du jour du dehors, du bleu du ciel, du blanc des rideaux, du jaune des murs tombant sur le bleu, le blanc et le gris, du costume de toutes ces créatures rangées et semblables, il résultait une certaine harmonie de tons rompus, dans une lueur répandue, diffuse et sans éclat, qui rappelait la tonalité d'un Chardin et le jour sobre et uni de ses intérieurs ${ }^{33}$.

De façon plus nette encore, la visite d'un hôpital (18 décembre 1860, I, 644), programmée à des fins romanesques ("Il nous faut faire, pour notre roman de Soeur Philomène, des études à l'hôpital sur le vrai, sur le vif... "), n’avait pas donné lieu à une description véritable du cadre, mais suscitera la scène de la ronde de nuit, inspirée de Rembrandt, dans l'ouverture du roman. Les Goncourt soulignent d'ailleurs dans le Journal leur incapacité à voir cette " réalité brute" ("Ce soir, il nous reste de tout cela une lointaine vision, quelque chose qu'il nous semble avoir rêvé, plutôt que vu") comme ce sera le cas pour ces autres lieux atypiques du Journal, l'hôpital où meurt leur servante Rose et l'amphithéâtre où est déposé son corps ${ }^{34}$. Mais cet autre lieu du drame de la servante, le cimetière, sera quant à lui minutieusement évoqué, parce qu'esthétisé, et le travail de l'aquarelle accompagnera la transcription écrite du Journal, que retravaillera ensuite le roman.

Il semble donc que l'investissement descriptif, même s'il change de teneur au début des années 1860, en devenant plus documentaire, répondant ainsi à une volonté de consigner la réalité, reste profondément lié à une finalité esthétique. Lorsque celle-ci est ab-

33. Journal, 28 octobre $1862, \mathrm{I}, 870$.

34. Journal, 17 août 1862, I $845 ; 20$ août 1862, I, 847 (c'est aussi l'émotion qui empêche alors de voir). 
sente, le lieu, rapidement évoqué, laisse la place à l'humain (il est symptomatique qu'Edmond se soit d'ailleurs servi de la notion de " document humain " pour théoriser a posteriori, en 1879, leur pratique romanesque dans la préface des Frères Zemganno). On ne trouve chez les Goncourt, ni cette absorption dans l'objet, même le plus bourgeois, comme un insondable qui amène une sorte de suspension dans le récit flaubertien, ni cette exhaustivité descriptive à des fins cognitives ou didactiques, comme chez Zola. La valeur plastique est une motivation fondamentale de la description chez les Goncourt.

Cela explique les choix opposés que formulent d'une part les deux frères dans les années 1860, d'autre part le seul Edmond dans les années 1880. Dans un cas, défiance à l'égard de la description du décor, parce qu'elle relève trop de la peinture, et étoufferait les personnages du roman; dans l'autre, nécessité de la description des réalités matérielles, dans une perspective esthétique désormais ouvertement assumée.

\section{La place de la description}

Les propos théoriques et critiques du Journal sur la place de la description dans le dispositif romanesque viennent à la suite d'une réflexion sur la matérialité en peinture, menée les années 1850 , et de l'effort pour assigner à chaque art son domaine propre ${ }^{35}$. Les Goncourt condamnent "la description pour la description », en recourant à des critères comme la "matérialité ", "l'empâtement ", la " grosse couleur », l'importance excessive du " détail ». Les choses l'emportent sur les hommes, le matériel sur le psychologique, le pictural sur le littéraire :

Au fond et dans le vrai, Madame Bovary - un chef-d'œuvre dans son genre, le dernier mot du vrai dans le roman - représente un côté très matériel de l'art de la pensée. Les accessoires y vivent autant et presque au même plan que les gens. Le milieu des choses

35. Voir mon étude Les Goncourt et la collection, op. cit., p. 259 sq. 
y a tant de relief autour des sentiments et des passions, qu'il les étouffe presque. C'est une œuvre qui peint aux yeux bien plus qu'elle ne parle à l'âme. La partie la plus noble et la plus forte de l'œuvre tient beaucoup plus de la peinture que de la littérature. C'est le stéréoscope poussé à sa dernière illusion ${ }^{36}$.

On peut mesurer la cohérence de la réflexion au début des années 1860, avec cette série de critiques convergentes adressées à ces grands praticiens de la description que sont Flaubert, et avant lui Balzac et Gautier, modèles reconnus des deux frères ${ }^{37}$. On comprend alors cette mise au point théorique à laquelle aboutissent les Goncourt :

La description matérielle des choses et des lieux n'est point dans le roman, telle que nous la comprenons, la description pour la description. Elle est le moyen de transporter le lecteur dans un certain milieu favorable à l'émotion morale, qui doit jaillir de ces choses et de ces lieux ${ }^{38}$.

Et Edmond de Goncourt rappellera plus tard comment l'effort des romanciers débutants ${ }^{39}$ pour s'affranchir de leurs modèles a porté d'une part sur le style, et d'autre part sur la place du descriptif :

Dans cette concurrence fraternelle à bien écrire, il était arrivé que mon frère et moi avions cherché à nous débarrasser de ce que nous devions à nos aînés : mon frère à rejeter le papillotage du style de Janin, moi la matérialité du style de Gautier. [...] Et surtout, il nous venait une horreur des grosses colorations, auxquelles j'avais un peu trop sacrifié, et nous cherchions, dans la peinture des choses matérielles, à les spiritualiser par des détails moraux ${ }^{40}$.

Après 1870 , on voit à l'inverse apparaître dans le Journal, toujours en relation avec le genre romanesque, des notations exclusi-

36. Journal, 10 décembre 1860, I, 642. Voir aussi 6 mai 1861, I, 692.

37. Journal, 6 octobre 1861, I, $736 ; 2$ novembre 1863, I, 1026.

38. Journal, 23 juillet 1865, I, 1176.

39. Cc qu'ils sont encore au début des années 1860 , où après leur bref passage dans le journalisme, ils n'ont encore écrit que des livres d'histoire.

40. Journal, 26 décembre 1895, III, 1210-12. 
vement descriptives, des éléments de décor intérieur beaucoup plus précis : "Le joli et curieux intérieur, pour un romancier, que la chambre de Mme de Girardin! Cette chambre, elle l'a fait non tendre, mais comme elle dit, "habiller" de satin brodé par Worth [... $~^{41}$. En 1881, c'est une dizaine de loges d'acteurs et d'actrices du Théâtre-Français qu'Edmond de Goncourt décrit, en vue de «la construction de la loge de la Faustin " ${ }^{42}$. Peu après, il fait, il est vrai à propos de Saint-Simon, et donc du genre différent des mémoires, un constat absolument inverse à celui qui avait suscité dans les années 1860 la critique de Balzac, de Gautier, de Flaubert: "Dans Saint-Simon, à la peinture, à l'admirable peinture des personnages, manque malheureusement la peinture des choses $"{ }^{43}$. D'autres remarques montrent bien que la description est désormais perçue comme une composante essentielle de la littérature, et un acquis du roman moderne (ce que souligne, et déplore, à la même époque, un critique comme Brunetière $\left.{ }^{44}\right)$ :

[...] l'on cause [chez Daudet] de littérature, de ses modes, de sa fabrication, et je dis que dans tous les romans anciens, on ne sait pas où ça se passe et qu'il n'y que dans les romans de ce temps où l'on perçoit le milieu d'une action romanesque, où l'on sait qu'on est en Orient ou en Occident, en France ou en Espagne, à Paris ou en province, au faubourg Saint-Germain ou au faubourg SaintHonoré, et cela par la vertu de la description qu'on a tant blaguée $e^{45}$.

Pour Edmond de Goncourt, qui revendique le titre de "descripteur " ${ }^{46}$, l'art de décrire est tributaire du regard, et de la capacité à percevoir la beauté artistique :

41. Journal, 24 avril 1872, II, 509.

42. Journal, 15 juin 1881, II, 897.

43. Journal, 11 août 1883 , II, 1017.

44. Le Roman naturaliste, 1878, "L'érudition dans le roman" (1877), p. 53. Voir aussi "Le roman réaliste en $1875 *(1875)$, p. 23.

45. Journal, 11 mai 1892, III, 706.

46. Voir cette remarque exaspérée du Journal: " je passe dans le public d'à présent pour un descripteur bien inférieur à cet ex-petit pion [Bourget]" (11 mai 1890, 111, 424). Quelques années avant, il avait pris soin de noter que Daudet voyait en lui un " homme de l'art ", un " voyeur de choses" (24 mai 1883, II, 1007). 
Au fond, ces lettrés [Zola, Daudet, Huysmans, Céard, Charpentier] n'ont d'yeux ni pour un bibelot ni pour un joli arbuste, ni pour quoi que ce soit arrêtant de force le regard de l'artiste. Et moi, qui suis sensible même à la beauté de couleur d'un morceau de terre retournée dans mon jardin, je ne serais pas supérieur à ces aveugles? C'est pas possible, vraiment - et dans une littérature devenue toute descriptive ! [18 avril 1883, II, 1000-1001].

La peinture d'intérieur est même considérée comme un type de description spécifique, qui s'impose dans des genres comme la chronique, et réclame en amont une compétence propre (savoir reconnaître et apprécier les objets d'art, débusquer le faux) ; pas de bon descripteur qui ne soit aussi connaisseur :

Hier, il est venu chez moi le reporter Puech de L'Éclair à l'effet de me peindre dans mon intérieur. Je crains bien que l'article ne soit pas si caressant que celui qu'il a consacré à Zola, car je n'ai pu lui cacher un mépris allant presque jusqu'au dégoût. Un monsieur qui fait profession de peindre des intérieurs et qui ne sait pas distinguer un dessin d'une gravure, une poterie japonaise d'une majolique, une commode de Riesener d'une commode du faubourg Saint-Antoine, enfin qui ne se doute pas de l'originalité, de la canaillerie ou du plagiat d'un mobilier ! (6 juillet 1890 , III, 444$)^{47}$.

Si bien que l'ambition littérairc d'Edmond de Goncourt, en ses dernières années, semble être devenue exclusivement descriptive :

Ah ! si j'avais encore quelques années à vivre, je voudrais écrire sur l'art japonais un livre dans le genre de celui que j'ai écrit sur l'art du XVIII' siècle, un livre moins documentaire, mais un livre encore plus poussé vers la description pénétrante et révélatrice des choses [25 mai 1888 , III, 127].

On voit donc bien la place de premier plan finalement conquise par la description, et particulièrement par celle du décor intérieur, devenue une priorité au-delà des frontières génériques. On va voir qu'elle occupait déjà chez les Goncourt une place importante dans

47. On voit ici comment l'accusation de plagiat fréquemment adressée ailleurs à Zola romancier est reportée de l'écriture aux objets collectionnés. Par ailleurs, notons comment on en revient à "l'intérieur " comme désignation du lieu privé. 
le texte d'histoire, place qui s'est peu à peu étendue, à mesure que la forme romanesque est remise en cause et que d'autres genres s'affirment, comme le texte d'histoire, le journal intime, les mémoires.

\section{Le lieu comme document historique Le lieu typique : pour une "physiologie" des lieux}

Au delà des notations descriptives à finalité romanesque, ou des propos plus théoriques sur la description dans le Journal, il faut considérer celui-ci comme texte d'histoire, peinture des mœurs du présent, et voir notamment la place qu'y occupe un lieu spécifique, "l'intérieur » au sens restreint d'habitation privée.

À mesure que l'écriture du Journal progresse dans le temps, il semble que les portraits et les types d'hommes (les "deux cousins", Timoléon de Villedeuil et Léonidas Labille, "deux pôles sociaux résumés en types: le républicain et le légitimiste " ${ }^{48}$ ) laissent peu à peu la place à des types de lieux. Le portrait de Léonidas en 1858, qui est précédé, comme on l'a vu, de la description minutieuse de son cabinet de travail, conclut sur cette généralisation : "C'est un cabinet à égorger de l'argent, la bauge où se tient tapie la lignée des acheteurs de biens nationaux $[\ldots] "$ ".

Cette vocation typique du lieu sera théorisée et exemplairement illustrée en 1862, avec une série de trois intérieurs d'hommes de lettres ${ }^{50}$. C'est une étape importante dans l'évolution des descriptions de lieux dans le Journal, puisqu'on a désormais affaire à un texte assez long et détaillé, et pourtant il ne porte pas sur des demeures d'amis ou de proches, comme c'était le cas pour la chambre de Blanche Passy ou le cabinet de Léonidas Labille. S'inspirant certainement de ces premiers essais de descriptions "longues", il prend aussi la suite de commentaires plus critiques, mais jusqu'alors expéditifs, sur des intérieurs d'hommes de lettres (Janin, Uchard,

48. Journal, 1852, I 38-40.

49. Journal, 26 septembre 1858, I, 402-404.

50. Journal, 27 novembre 1862, I, 890-891. 
Dennery, Scholl $\left.{ }^{51}\right)$, courtes mentions descriptives déjà "orientées" par un jugement de valeur, qui ont jusqu'alors prévalu (et sur lesquelles on aura l'occasion de revenir pour expliquer, en termes de stratégie sociale, la motivation qui les fonde).

Pour théoriser la valeur typique du lieu, les Goncourt se réferent explicitement au genre des physiologies, que les Goncourt débutants ont eux-mêmes pratiqués .

En vertu du lien qui les unit (et qui n'est pas ici développé, mais il semble que ce soit plus l'homme qui choisit et modèle le lieu, plutôt que le lieu qui conditionne et détermine l'homme, comme le diront plus tard Taine et Zola), le lieu devient représentatif de son occupant.

Mais, comme l'exemple du cabinet de Léonidas le montrait déjà, la description du lieu débouchant finalement sur le portrait de l'occupant, c'est bien l'évocation de la personne qui légitimise la description du décor. Les trois intérieurs d'hommes de lettres sont bien des portraits sur le modèle de La Bruyère, ils aboutissent au dévoilement d'un personnage :

On monte, rue Jacob, au fond d'une cour, cinq étages. On suit un corridor $[\ldots]$. On frappe $[\ldots]$. On est dans une sorte de belle chambre de cocher, pleine de livres en désordre sur le carreau. Une voix, de l'autre pièce, crie comme du fond d'un rêve : "Qui est là ?" On entre, on voit une chambre de grisette, d'ouvrière couturière, une table de nuit où il $\mathrm{y}$ a beaucoup de livres [...]. Les livres sont neufs : vous êtes chez un critique en chambre. C'est Montégu, qui de la Revue des Deux Mondes, vient de passer au Moniteur.

Si la description du lieu débouche sur l'évocation de l'occupant, on peut néanmoins noter la place qu'occupe le décor - à la différence de La Bruyère où c'est essentiellement l'apparence physique, le comportement qui servent à brosser le portrait, à la différence

51. Journal, 18 octobre 1857, I, 301 (Uchard) ; 24 janvier 1860, I, 521 (Janin) ; 11 mars 1860, I, 541 (Dennery) ; 18 octobre 1860, I, 624 (Scholl), etc.

52. La Cuisinière, envoyée à l'éditeur Curmer, à en croire Edmond (Journal, 18 mars 1885, II, 1145), et surtout La Lorette, publiée en plaquette en 1853 (rééditée en 2002, Du Lérot, par A. Barbier Sainte Marie). 
aussi des physiologies où le lieu est une composante plus fréquente mais non indispensable. Ici, le portrait physique de l'homme est rapide, et ne constitue qu'une étape dans le processus d'identification, jusqu'au dévoilement du nom.

Rue d'Argenteuil [...] un escalier noir [...] un salon où il y a des pauvres meubles d'une élégance maigre [...]. Dans un coin, un piano [...]. Les rideaux sont maigres aux fenêtres [...]. Là-dedans un petit homme maigre, très maigre, très pâle, des yeux de fouine, des cheveux rares, mais longs, un teint de papier : c'est Édouard Fournier, le critique à tout faire de La Patrie.

En outre, on peut noter comment la maison s'impose ici comme un modèle pour penser la société, du moins comme une image pour la représenter : "Cela [l'homme de lettres] loge généralement en haut, au cinquième: Paris a le cerveau comme l'homme, en haut. Ce qui court est en bas, boutiques, entresols ; ce qui digère au premier : la maison est individu ". De façon assez proche - conséquence probable du rôle croissant qu'ils accordent aux lieux dans l'écriture du Journal -, les Goncourt écrivent peu après : "Hier, nous étions dans le salon de la princesse Mathilde. Aujourd'hui, nous sommes dans un bal du peuple, à l'Élysée des arts, au boulevard Bourdon; j'aime ces contrastes. C'est monter la société comme les étages d'une maison ${ }^{53}$."

Les années 1860 représentent dans le Journal la grande décennie des descriptions de lieux, des "physiologies par les lieux ». Ensuite, on aura des évocations de lieux moins abondantes, mais beaucoup plus détaillées, et surtout "autonomisées", qui constitueront non plus des physiologies par le lieu, mais des physiologies de lieux au sens propre : l'occupant, l'habitant n'y seront plus décrits, et Edmond indiquera bien que le lieu a été observé pendant son absence. Dans ce cas, la stratégie sociale, sensible dans la valorisation (ou la dépréciation) du lieu, pourra s'être effacée, puisqu'il ne s'agira plus, à partir du lieu, d'agir sur l'homme. La description, plus neutre, aura surtout cette valeur historique de consigner une

53. Journal, 9 février 1863, I, 931. 
mode, une façon d'habiter: "Elles sont, ces loges [des actrices du Théâtre-Français] un curieux symptôme du goût, du mobilier et du rococo des années présentes $"{ }^{54}$. En outre, la description pourra être détachée de tout projet, et venir simplement meubler le loisir du scripteur. Ce qui est alors remarquable, c'est la façon dont elle est annoncée, et comme telle, beaucoup plus nettement découpée, cadrée. Edmond évoque son attente dans le lieu vide, et le temps ainsi laissé au plaisir de voir et de décrire :

Aujourd'hui, je vais acheter des arbustes chez Moser à Versailles. Il est dehors et me fait attendre, et n'ayant rien de mieux à faire, je m'amuse à prendre la description de son cabinet, le cabinet d'un horticulteur de la banlieue de Paris ${ }^{55}$.

Dans les ouvrages des Goncourt consacrés au XVIII siècle, on trouve confirmation du rôle essentiel qu'ils accordent à la description de lieu dans une perspective historique. Le lieu, cadre de vie, est l'écrin du passé ; par l'évocation détaillée du lieu, le passé se ranime, et c'est pourquoi la description s'appuiera prioritairement sur l'image :

[...] pour entrer dans la société du XVIII siècle, pour la toucher du regard, ouvrons un carton de gravures, et nous verrons ce monde, comme sur ses trois théâtres, dans le salon de 1730 , dans le salon de 1760, dans le salon de 1780. / Ici, dans le premier salon, le monde est encore en famille [...] Dans la pièce large et haute, entre ces murs où les tableaux montrent des baigneuses nues, sur les ramages des panneaux de soie, sur les lourds fauteuils aux bras, aux pieds tordus, près de cette cheminée où flambe un feu clair et d'où monte la glace sortant d'une dépouille de lion et couronnées de sirènes $[\ldots]^{36}$.

En outre, ce texte, qui explicite sa démarche (passer par le lieu pour ressusciter les hommes, par le cadre physique pour redécouvrir la

54. Journal, 15 juin 1881, II, 897. Mais ces loges sont aussi décrites en vue du roman de l'actrice (La Faustin).

55. Journal, novembre 1880, II, 874 . Voir aussi 10 juin 1882 , II, 945 , et 2 juin 1889 , III, 275 : "Donc j'attends et je me mets à étudier cet intérieur [Perrin, fils du directeur de l'Opéra], qui peut être considéré comme l'intérieur du mondain artiste ".

56. La Femme au XVIII siècle, ouverture du chap. II. 
société), se présente comme une typologie (des époques par les lieux), et la tripartition annoncée d'emblée évoque irrésistiblement les " trois intérieurs d'hommes de lettres " du Journal, qui datent de la même année 1862 :

Trois époques apparaissent dans la société du XVIII siècle. Trois évolutions de son histoire attribuent trois formes à son esprit social et lui imposent trois modes. Le commencement du règne de Louis $\mathrm{XV}$, la fin de ce règne, le règne de Louis XVI apportent au monde qu'ils transforment et renouvellent successivement le changement de trois âges. Et c'est la physionomie de ces trois âges qu'il faut étudier d'abord.

La convergence méthodologique entre le texte du Journal et cet extrait de La Fermme au XVIII siècle est flagrante. Elle montre que le Journal est bien un texte d'histoire du présent, que l'attention aux lieux est centrale dans cette perspective historicisante, et comment les Goncourt l'ont théorisée et systématisée autour de $1862^{57}$.

\section{Le lieu singulier, le lieu historique}

Il faut encore approfondir l'analyse de la démarche historique dont relève le Journal, car elle implique au moins deux perspectives différentes. Le plus souvent, les Goncourt adoptent la posture du peintre de mœurs, qui est celle de la proximité et de la fidélité au présent dans son détail ; mais dans d'autres cas, ils se placent sous le regard de l'Histoire, ils écrivent des mémoires, ils soulignent la dis-

57. On voit bien comment le texte d'histoire et la médiation de l'image autorisent (et sollicitent) le déploiement de la description de lieu. Voir aussi la rivalité qui s'établit entre description d'après l'image et description d'après nature, qui renvoie probablement à une opposition générique, entre le livre d'histoire et le roman, histoire du présent, informée par le témoignage direct : "Je crois vraiment que lorsqu'on sait regarder, découvrir tout ce qu'il y a dans une image, on n'a pas besoin d'aller dans les pays à images. Ainsi, aujourd'hui, ayant sous les yeux une image de Toyokuni, représentant le bureau d'une Maison verte, d'une maison de prostitution, et me faisant donner une explication de tous les objets grands et petits garnissant ce bureau, $j$ 'avais la conviction que j'apporterais au lecteur, avec ma description, une sensation du rendu de l'endroit, tout aussi photographique que la donnerait une description d'après nature de Loti" (Journal, 4 avril 1891, III, 569). 
tance qui déjà les sépare de faits plus anciens, ou le recul qu'ils savent prendre pour mesurer l'importance d'un événement au regard de la postérité. À la fidélité qui nivelle, s'oppose alors la sélection qui isole. Or certains lieux sont considérés comme particulièrement dignes de mémoire, et décrits pour le rôle qu'ils ont pu jouer dans l'histoire culturelle, l'histoire littéraire :

Comment ce cabaret où a passé la bohème n'a-t-il pas été l'objet d'une description, n'a-t-il pas été célébré dans un livre ?

Un cabaret dans un terrain vague de Vaugirard, à l'entrée des carrières, devenues des champignonnières, et tout étincelant de beaux cuivres, de reflets de bouteilles aux formes trapues, d'un tas de vieilleries bien luisantes, qui semblaient le mobilier retrouvé d'une auberge de l'ancienne France [...].

Oui, c'était, ce cabaret, le cabaret du frère de Bonvin $[\ldots]^{58}$.

Ces lieux ont en eux-mêmes une valeur historique, au moins égale à celle de leur occupant, comme c'est le cas pour l'atelier de la Princesse Mathilde :

Dans l'atelier, je suis seul, désœuvré, et un blanc soleil éclaire si joliment toutes les choses qui sont là, qu'il me prend la tentation de les décrire. Je veux laisser un souvenir de cette pièce, qui fut vraiment, pendant l'Empire, l'aimable domicile du gouvernement de l'art et de la littérature, le gracieux ministère des Grâces. Je veux laisser un souvenir ressemblant à la fois à une peinture et à un inventaire de commissaire-priseur, quelque chose qui, dans les temps futurs, permette à ceux qui aimeront la mémoire de la Princesse, de la retrouver, de la voir, comme s'ils poussaient la porte de son atelier, gardé dans la cendre d'une Pompéi".

On voit bien par cette ultime comparaison comment Edmond de Goncourt se place sous le regard de l'Histoire, par une prise de distance qui accuse les reliefs, qui fige le lieu et le préserve en le soustrayant au temps.

58. Journal, 5 octobre 1879, II, 845. Le lieu est (encore) décrit comme un tableau de Chardin, un des maîtres précisément de Bonvin, et la motivation esthétique rejoint ici la légitimation historique de la description.

59. Journal, 14 novembre 1874 , II, 606. 
Les lieux décrits par le Journal comme historiques le sont cependant de manière paradoxale : importants pour l'Histoire, mais suffisamment modestes, ou réservés, ou du ressort de l'intime (reflétant la personne, non dans ce qu'elle a de typique, mais dans ce qu'elle a d'individuel), pour n'être pas connus du plus grand nombre. S'impose ainsi la nécessité, pour le Journal, d'en rendre compte. A la croisée du singulier, de l'historique et de l'intime, viendra $s^{\prime}$ inscrire la propre demeure d'Edmond de Goncourt ${ }^{60}$. En 1874, la description de l'atelier de la princesse Mathilde (complétée un peu plus tard d'une évocation de sa chambre à coucher, et riche aussi d'évocations antérieures d'autres parties de la demeure), annonce bien la Maison d'un artiste $(1881)^{61}$.

\section{La stratégie sociale de valorisation du lieu}

La physiologie des lieux que met en place le Journal au cours des années 1860 est au service de l'histoire, mais relève aussi d'une stratégie de positionnement social : l'effort pour ranger dans une typologie le lieu visité, et particulièrement l'intérieur, le lieu privé, est souvent un moyen de lui faire perdre sa spécificité, de désamorcer l'impact ou le prestige du singulier. Le discours sur le lieu est ainsi une façon d'avoir prise sur l'homme : plus qu'une clé qui aide à le comprendre, le décor est aussi un cadre qui le contient, et aide à démythifier les grandes figures, proches des sphères du pouvoir

60. On comprend inversement le discours négatif que suscitent toujours ces lieux "semi-publics" que sont les restaurants (voir notamment l'article de Joëlle BonninPonnier, Cabiers Goncourt, $\mathrm{n}^{\circ}$ 9, 2002) et surtout les chambres d'hôtel (Journal, septembre $1861, \mathrm{I}, 731 ; 29$ décembre 1865 , I, 1217 ; 28 juillet 1867 , II, 99-100; 16 août 1868, II, 170).

61. Outre sa valeur historique et personnelle, l'atelier de Mathilde vaut parce qu'il fait image, parce qu'il est "peinture " (après l'indication de lumière initiale, les qualités plastiques du lieu éclatent à tout moment: "tout un monde de choses, où brillent les reflets des métaux, où éclatent les couleurs des plumages exotiques"). Ce passage, par sa double ambition (historique et esthétique), sa démarche (description minutieuse à partir d'une présentation du dispositif sparial - les façades, le lien entre la maison et l'atelier, le tour de la pièce en suivant les murs, et en respectant l'agencement décoratif de chaque mur en "panneau ") et sa relative longueur ( 4 p.), préfigure véritablement la Maison d'un artiste. 
(de la maison de la princesse Mathilde à Saint-Gratien, les Goncourt disent bien qu'elle " n'a rien d'un palais. L'intérieur n'a rien de princier $"{ }^{62}$ ), à reconsidérer les grands noms (actrices comme Rachel $^{63}$, comme Eugénie Doche, dont l'appartement, écrit Jules, se révèle "moins somptueux en somme que je ne pensais " ${ }^{64}$ ). Inversement, l'ami Chennevières, devenu après 1870 Directeur des Beaux-Arts, est resté accessible : à preuve son intérieur qui n'a pas changé ${ }^{65}$.

On aura donc affaire à une typologie paradoxale, et axiologiquement orientée : au lieu de dégager des différences, elle s'efforce de les atténuer, et réunit dans la même critique des lieux pourtant très distincts. Les intérieurs d'hommes de lettres sont au mieux des logements "d'ouvriers des lettres", au pire, et le plus souvent, caractéristiques de ce néant du style qu'on pourrait appeler le "putain-canaille" (a contrario la Maison d'un artiste montrera le décor singulier dont a su s'entourer un véritable homme de lettres). Janin habite " un chalet capitonné, qui ressemble à un cottage de vieille putain, meublé de cadeaux de gens de mauvais goût, le rêve d'un bourgeois d'opéra-comique..." " fille " ${ }^{67}$; Sainte-Beuve, "un salon froid, nu, bourgeois et banal, rappelant assez le salon d'une maison de prostitution d'une ville de province " (on voit avec quelle aisance les Goncourt rabattent le lieu bourgeois sur le lieu vulgaire, caractérisé par la prostituée ${ }^{68}$; Feydeau, " un appartement de fille et d'artiste, un luxe de bibelots malsains, une artisterie de boursier, pour ainsi dire, avec je ne sais quoi qui sonne faux et qui semble louche, qui sent l'hommeputain " ; quelque vingt ans plus tard, Maupassant a lui aussi " un

62. Journal, 16 août 1862, I 842. Voir aussi Véron, 3 décembre 1861 , I 755 ; le préfet de police Boitelle, 29 juin 1863, I, 982 ("Le cabinet du préfet de police, cette grande pièce, ces murs où s'agitent tant de choses redoutables, frappés de tant de secrets hideux ou terribles [...] tout cela est rempli de Boucher [...]").

63. Journal, 11 avril $1858, I, 341$.

64. Journal, 13 décembre 1858, I, 427.

65. Journal, 22 février 1874 , II, 570.

66. Journal, 24 juin $1860,1,521$.

67. Journal, 24 août 1860, I, 591.

68. Journal, $1^{\text {er }}$ décembre $1862, \mathrm{I}, 893$.

69. Journal, 24 juin 1863, I, 978. 
mobilier de putain ${ }^{70}$. Même lorsque, pour un même individu, les logis successifs sont symptomatiques d'une ascension sociale, les deux frères semblent n'enregistrer qu'une permanence dans la laideur et le mauvais goût : ils gomment l'évolution, comme ils effacent la spécificité. Ainsi Scholl, que ce soit rue de "Rue de LaTour-d'Auvergne", dans un "rez-de-chaussée humide dans une maison appartenant aux hospices", ou "rue Laffitte, à côté de la Maison d'Or ", habite-t-il toujours, du début à la fin des années 1860 , un "appartement de putain et de journaliste "

A partir des années 1880 , la valorisation positive succède à la critique, quand Edmond de Goncourt ne juge plus ses pairs, mais ses disciples et ses émules. Il procède pourtant toujours au nivellement des différences : ils habitent tous, à l'en croire, un petit logement fleuri, un cottage anglais ${ }^{72}$. Hennique et son " petit intérieur gentiment arrangé, avec de la japonaiserie bon marché " ${ }^{73}$, Bonnetain dans son " petit corps de logis ", "égayé avec de la japonaiserie à bon marché " ${ }^{74}$, nullement raillés pour cette décoration modeste (qui déclenche ailleurs les sarcasmes du spécialiste qu'est Edmond de Goncourt), rejoignent Ajalbert et son cinquième de l'avenue Bugeaud, "petit intérieur tout fleuri de pivoines et d'iris " ${ }^{75}$, Mirbeau et sa maison de Carrières-sous-Poissy, un "intérieur d'après le modèle des cottages anglais, toujours égayé de couleurs claires ${ }^{76}$. De même, pour les peintres, Raffaelli dans son " nouveau logis, gentiment arrangé à la façon d'un cottage anglais " 77 , Forain dans son "petit intérieur ", "ingénieusement machiné à l'anglaise "

70. Journal, 18 décembre 1884, II, 1121, et 25 mars 1890, III, 405.

71. Journal, 18 octobre 1860, I, 624; 10 décembre 1860, I, 640; 6 mai 1862, I, 814 ; 9 décembre 1866, II, 54 .

72. Similitude qui est également la marque d'une transformation des modes décoratives, comme me le rappelle Pierre Dufief. Edmond de Goncourt détaille par exemple le "papier anglais à fond blanc tout fleuri de bouquets d'œillets à l'imitation des toiles de Perse " dans l'appartement parisien de Mirbeau, avenue de l'Alma (6 juillet 1895, III, 1150).

73. Journal, 5 avril 1890 , III, 410.

74. Journal, 12 février 1888, III, 96.

75. Journal, 15 juin 1895, III, 1143.

76. Journal, 6 juillet 1895 , III, 1150.

77. Journal, 9 avril 1892, III, 690.

78. Journal, 21 mai 1892, III, 714. 
Au-delà de la fonction discriminatoire qu'acquiert l'intérieur privé, et qui permet de positionner son occupant dans l'espace culturel et social tel que le redessine le Journal, il faut noter l'ambiguïté de certains jugements de valeur. Si les demeures d'actrices (Rachel, Eugénie Doche, Suzanne Lagier, et même, quelque trente ans plus tard, Sarah Bernhard ${ }^{7}$ ) sont souvent la cible de jugements sévères, à l'inverse, la loge d'actrice est presque toujours positivement connotée (voir notamment « la coquette loge de Réjane, aux murs comme habillés de ruban et aux meubles de laque blanc ${ }^{80}$ ); en outre, elle présente des similitudes importantes avec la pièce du cabinet de toilette de cette demeure modèle de l'écrivain qu'est la Maison d'un artiste ${ }^{81}$, pièce également valorisée quand Edmond décrit dans le Journal d'autres demeures d'écrivains, dont la décoration d'ensemble sinon lui déplaît. Ainsi de Zola (une habitation "déshonorée par un épouvantable mobilier", mais un "somptueux cabinet de toilette " ${ }^{82}$ ), de Montesquiou ("Un logis tout plein d'un méli-mélo d'objets disparates, de vieux portraits de famille, d'affreux meubles de l'Empire, de kakémonos japonais, d'eaux-fortes de Whistler. / Une pièce originale : le cabinet de toilette $[\ldots] »)^{83}$.

On constate un semblable retournement à propos des demeures de courtisanes. Les Goncourt en déplorent systématiquement le luxe voyant et le mauvais goût (le "gros luxe d'impure " de la Deslions ${ }^{84}$, les "glaces du plus riche mauvais goût $\mathrm{XVI}^{\mathrm{e}}$ siècle " de la Barrucci $^{85}$, l'« appartement de courtisane de théâtre, avec des accessoires dorés " de Jeanne de Tourbey ${ }^{86}$, "la salle à manger qui, avec tout son luxe et la surcharge de son mauvais goût Renaissance, ne ressemble guère qu'à un très riche cabinet de grand restaurant "

79. Journal, respectivement 11 avril 1858, I 340-341; 13 décembre 1858, I, $427 ; 27$ février 1863, I, $940 ; 17$ octobre 1893, III, 878.

80. Journal, 22 mars 1892, III, 684. Voir aussi la loge de Lia Félix (22 décembre 1859, I, 497 ; 22 janvier 1860, I, 519); les loges du Théâtre-Français (15 juin 1881, II 897).

81. Voir la réédition récente, Dijon, L'Échelle de Jacob, 2003, t. II, p. 189 sq.

82. Journal, 2 avril 1891, III, $568 ; 7$ mars 1894, III, 927 ; 25 février 1896, III, 1242.

83. Journal, 7 juillet 1891 , III, 604 .

84. Journal, 16 mars 1862, I, 789.

85. Journal, 8 novembre 1863, I, 1026.

86. Journal, 16 avril 1865, I, 1155. 
chez la Païva ${ }^{87}$, et chez la Leininger, "partout un grand luxe, mais un luxe commun et acheté tout d'un coup ${ }^{88}$ ). Et pourtant, c'est la maison de Jeanne de Tourbey qu'ils faillirent acheter pour euxmêmes en 1868 ; et non seulement ils en déplorent la perte en des termes qui témoignent d'un fort investissement affectif, mais ils font aussi intervenir pour la décrire un paradigme qui sera récurrent dans La Maison d'un artiste, celui de la "petite maison ", lieu galant du XVIII siècle $^{89}$ :

[...] une maison bizarre, presque cocasse, ressemblant à une petite maison d'un sultan de Crébillon fils, mais qui nous a charmés, ensorcelés, par le je ne sais quoi de son originale étrangeté. Elle nous plaît sans doute parce qu'elle n'est pas la maison bourgeoise de tout le monde. Avec cela, un beau jardin, de vrais arbres. Et nous voilà tout le jour dans un contentement anxieux et dans une fièvre de rêves, suspendus sur cette pensée de maison $[\ldots]^{90}$.

Par ailleurs, notons qu'Edmond s'inspire explicitement de la Païva pour la décoration de son petit salon d'Auteuil. Dans $L a$ Maison d'un artiste, il ne craint pas de la citer, et de dire qu'il lui doit le choix original d'une tenture rouge pour mettre en valeur ses objets d'art.

Au-delà des valorisations diverses des intérieurs évoqués dans le Journal, un imaginaire de la maison, une esthétique et une écriture du lieu intérieur se mettent peu à peu en place au long de ces années 1860 , se nourrissant de toutes les expériences, de tous les lieux visités, même si ceux-ci semblent récupérés en surface par d'autres types de discours, plus polémiques" ${ }^{91}$ Des critères constitutifs de l'intériorité matérielle émergent (féminité, sensualité, esthéticité), auxquels il manque encore l'épaisseur historique et sémantique du

87. Journal, 24 mai 1867, II, 84.

88. Journal, 10 août 1875 , II, 657.

89. Voir notamment op. cit., t. II, p. 99-104 (long extrait de La Petite Maison de JeanFrançois de Bastide, 1763).

90. Journal, 22 et 26 juin 1858, II, 158.

91. C'est la preuve que le modèle normatif de l'homme de lettres collectionneur artiste selon les Goncourt procède non seulement par exclusion, mais aussi par assimilation. 
lieu, que le collectionneur saura quant à lui déployer dans sa demeure ${ }^{92}$.

\section{Le lieu comme modèle esthétique et matrice littéraire : la maison}

La motivation esthétique des descriptions de lieux est déjà apparue à plusieurs reprises ${ }^{93}$, elle semble associée à la plupart des discours que suscite l'espace intérieur, peut-être parce qu'elle est pour les Goncourt la légitimation la plus sûre de la forme descriptive, peut-être aussi en vertu d'une sorte de nécessité structurelle, les Goncourt ne concevant le lieu clos, habité, décoré par l'occupant, que comme cette enveloppe individuelle qui prédispose à la jouissance (sensuelle, ou esthétique, et l'on comprendrait alors ce qui rapproche la courtisane de l'homme de lettres) ${ }^{94}$. Un autre texte du Journal, qui n'est pas à proprement parler la description d'une maison, mais du mode de vie, du train de vie dans une demeure du XVIII ${ }^{e}$ siècle (Edmond cite et commente un livre ayant pour titre "La maison réglée ou l'art de diriger la maison d'un grand seigneur $\left.[\ldots]{ }^{95}\right)$, illustre bien, au-delà des qualités plastiques, le dis-

92. L'hôtel de la Païva est "un rêve de tapissier, sans un morceau de passé, sans un meuble, une statue, un tableau, qui sauve une maison de l'ennui du neuf et y met l'intérêt et l'amusant de l'historique " (Journal, 31 mai 1867, II, 87). A l'inverse, Edmond écrira plus tard : "Dans la pierre ancienne d'une vieille maison se lève chez vous un petit sentiment de jouissance qu'on n'a pas parmi du moellon d'hier, sentiment de jouissance très difficile à définir, mais parent du sentiment qu'on éprouve en voyageant dans un pays qui a un passé " (18 septembre 1894, III, 1011).

93. Voir aussi ces portraits féminins, où la femme semble un bibelot mis en valeur par le cadre (24 novembre 1884, II, $1117 ; 25$ avril 1891 , III, 576 : " un grand salon aux boiseries dorées, égayé par un admirable meuble de Beauvais aux bouquets de fleurs les plus papillotants sur un fond crème, un meuble au nombre incroyable de chaises, de fauteuils, de grands canapés, de petits canapés pour tête-à-tête [...] Là-dedans, au milieu de ce mobilier d'un autre siècle [...]").

94. On rejoint ici les analyses de Benjamin sur le dispositif culturel et mental qui, au XIX siècle, accorde tant d'importance à l'intérieur, c'est-à-dire au sujet, à la personnalité individuelle qu'il protège et qu'il libère (voir "Louis-Philippe et l'intérieur " dans Paris, capitale du XIX siècle, Cerf, 1989).

95. Journal, 6 avril 1894, III, 938-943. Notons en outre qu'Edmond a acquis l'ouvrage sur la foi de son seul titre, preuve supplémentaire de cette fascination pour la 
positif formel que thématise la maison, et qui repose tant sur une mise en ordre, que sur une dynamique complexe, la vie quotidienne dans la maison suscitant toute une série de mises en relation (de l'habitant à ses objets, des objets entre eux, etc.). De plusieurs façons donc, le lieu intérieur fonctionne bien comme un modèle esthétique.

On peut, en outre, envisager le rôle effectif qu'Edmond de Goncourt assigne au lieu intérieur dans l'engendrement de l'écriture. D'abord, parce que dans le Journal, qui accompagne, prépare et prolonge tous les projets d'écriture, la maison des Goncourt apparaît bien comme un actant essentiel lorsque Edmond enregistre les aménagements, les transformations successives de ce lieu intime et les sentiments qu'il éprouve à son égard - ils oscillent entre détachement ponctuel et affection marquée ${ }^{96}$. Ensuite, parce que le rôle dévolu à la maison d'Auteuil était préparé depuis longtemps dans le Journal par toute une série de rêves et de désirs de lieux ${ }^{97}$. Enfin, parce que le Journal a en commun, avec La Maison d'un artiste, une série de textes, reproduits, prolongés, ou imités, que réunit en outre

" maison "; en 1894, il prépare justement ce complément à La Maison d'un artiste qui figurera à la date du 14 décembre...

96. Journal, 14 juillet 1870 , II, 263 : “Cette maison où j'ai tant souffert, j'y suis attaché par un lien que je ne soupçonnais pas "; 25 août 1870 , II, $270 ; 13$ septembre 1874, III, 590 : "Ma maison même ne me semble plus être pour moi ce qu'elle était il y a six mois. Je ne sais quelle indifférence de mourant m'est venue avant l'heure " : 22 octobre 1874, II, 593 : "Jamais je ne suis rentré chez moi avec aussi peu d'intérêt pour ma maison, mon travail, les goûts de ma vie"; 30 novembre 1874, II, 613. Voir aussi la variété des jouissances esthétiques, l'escalier tout fleuri de chrysanthèmes ( 1 "r novembre 1885, II, 1194), le " trou lumineux " (et le lien bien établi entre ce dispositif spécifique et l'activité d'écriture, 13 septembre 1890 , III, 463).

97. Journal, 18 avril 1857, I 251 : "Je voudrais une chambre inondée de soleil [...]"; 25 octobre 1860, I, 628; 8 octobre 1861, I, 737 : "Entrer dans une maison, et en entrant dans cette maison, entrer dans son rêve, s'y promener, s'y asseoir, le toucher, le voir ! [...] Cettc maison m'est restée dans les yeux, dans la tête. Je n'ai jamais rien désiré de ma vie aussi passionnément qu'elle"; 14 novembre 1870, II, 340 : "Mais parmi toutes ces bâtisses, il en est une qui me parle, je ne sais pourquoi. [...]"; 26 octobre 1894, III, 1023 "Mon château d'Espagne [...]". 
la même perspective, puisqu'ils débouchent sur la mise en scène de l'acte d'écriture ${ }^{98}$.

Le premier de ces textes, est le passage du 14 février 1888 , où Edmond de Goncourt évoque les boutiques de brocanteurs du boulevard Beaumarchais, qu'ont remplacées des commerces plus modernes". C'est apparemment l'envers de La Maison d'un artiste, puisque la déambulation urbaine s'est substituée à la réclusion sédentaire du collectionneur. Mais c'est l'amplification d'une thématique qui apparaissait déjà dans l'ouvrage de 1881, car le catalogue des dessins du Petit salon était précédé d'une évocation des marchands d'autrefois. En outre, on retrouve dans les deux textes la même tonalité nostalgique du ubi sunt. Le règne de l'exhibition mensongère a succédé à l'authentique enfoui sous la crasse ("ces boulevards aux candides et sales boutiques de ferrouillats, ignorant encore la mise en scène et le montage de coup, par la brochure et la photographie, de l'objet d'art, montré sous un coup de jour, dans le clair-obscur d'un petit salon ad hoc"); d'où les déplorations du promeneur :

Bien rares, hélas! sont les noms connus du temps de ma jeunesse.

Qui peut reconnaître dans le remaniement de la bâtisse, l'endroit où était la boutique de Vidalenc, cet antre aux carreaux poussiéreux, à la ferraille infecte garnissant la margelle de la porte, et tout bondé à l'intérieur de trésors! Ah! les merveilles que j'ai vues là, et dans tous les genres, mais surtout quelles boiseries! quels lits à la duchesse, à la polonaise, à tombeau ! Quelles ottomanes! quels fauteuils à poches, à cartouches, en cabriolet, en confessionnal! Quelles chaises en prie-Dieu! Il semblait que ce magasin

98. La Maison d'un artiste renferme en son sein le cabinet de travail l'écrivain. Voir mon analyse dans les Goncourt et la collection, op. cit., p. 222 sq.

99. Journal, III, 97. Ce passage sera publié sous le titre "Une promenade au boulevard Beaumarchais " dans le collectif Les Types de Paris [textes de Daudet, Zola, Bourget, Maupassant, Mallarmé, Mirbeau, etc., illustrés par Raffaelli], Éd. du Figaro, 1889. Le tcxte de l'édition Ricatte est en fait celui, non du manuscrit, mais de la version imprimée de 1894 du Journal, postérieure donc à la publication des Types de Paris. Trois versions successives du même texte, qui soulignent bien son importance. 
fût le garde-meuble de tout le mobilier contourné et si adorablement sculpté du XVIII siècle $^{100}$.

Enfin la promenade, causée par la fermeture d'une bibliothèque (celle du musée Carnavalet), aboutit à une maison d'écrivain, elle aussi fermée; de sorte que ce qui encadre le passage, c'est bien la référence à deux lieux de l'écrit, également clos.

Et j'allais quitter le boulevard du temple, quand en face du Café turc, je m'arrêtai, un moment, devant le $\mathrm{n}^{\circ} 42$, la maison à la petite porte cochère basse, où demeurait autrefois Flaubert et où, dans les batailles de paroles et les violences du verbe, la spirituelle et crâne Lagier apportait une verve si drolatique, si cocasse, si amusante.

La maison, lieu mort, mais, dans le passé, lieu de la parole vive et de l'écriture. Le style de Flaubert est de fait pastiché par "l'ironie" et le "vide" de la phrase finale, que la l'inscription ultime, dénuée de sens, fait d'autant mieux résonner :

La maison n'a plus le sourire d'autrefois, son plâtre a vieilli, des persiennes fermées disent des appartements sans locataires, et dans une boutique du rez-de-chaussée, semblant avoir fait faillite, on lit sur une immense bande de toile, qui a l'air d'une ironie, au-dessus du local vide : Cabaret de la folie, Tout Paris voudra voir les bandits corses.

Autre passage du Journal, qui rencontre le texte La Maison d'un artiste et fait de la demeure le lieu par excellence où s'engendre l'écriture : celui du 30 août 1892 qui évoque les habitations successives de la tante Nephtalie ${ }^{101}$. À l'occasion d'une réédition de $\mathrm{Ma}$ dame Gervaisais, Edmond de Goncourt revient sur sa tante, modèle de l'héroïne, pour en brosser un portrait plus détaillé. Il cite notamment ce qu'il en a déjà dit dans La Maison d'un artiste ${ }^{102}$

100. Avec son rôle de réunion et de préservation, avec le vocabulaire spécialisé du meuble qu'elle suscite, on voit bien comment la boutique joue ici le rôle du musée, et fonctionne bien comme l'envers (ouverture, publicité, circulation marchande) de la maison du collectionneur.

101. Journal, III, 748.

102. Op. cit., t. I, p. 355 sq. 
(l'influence de la tante sur ses goûts de collectionneur), mais il l'insère dans une description beaucoup plus précise du personnage et particulièrement des demeures successives où il lui a rendu visite enfant. Le premier appartement est associé au souvenir des premières lectures et des premiers élans de l'imagination :

De cet appartement où j'ai vu pour la première fois ma tante, il ne me reste qu'un souvenir, le souvenir d'un cabinet de toilette à la garniture d'innombrables flacons de cristal taillé et où la lumièrc du matin mettait des lueurs de saphir, d'améthyste, de rubis, et qui donnaient à ma jeune imagination, au sortir de la lecture d'Aladin ou la lampe merveilleuse, comme la sensation du transport de mon être dans le jardin aux fruits de pierre précieuse ${ }^{103}$.

Du deuxième appartement demeure le souvenir d'un dîner avec Rachel (" un dîner où le talent de la grande artiste dramatique était pour nous seuls et où je me sentais tout fier et tout gonflé d'être des convives "). Et de l'ancienne petite maison que Nephtalie occupe à Ménilmontant, Edmond retient surtout la "salle de spectacle ruinée, devenue une resserre d'instruments de jardinage ", où le dramaturge en herbe assiste aux représentations de son imagination :

[...] une salle aux assises des places effondrées, comme en ces cirques en pleine campagne de la vieille Italie, et où je m'asseyais sur les pierres disjointes et où je passais des heures à regarder, dans le trou noir de la scène, des pièces qui se jouaient dans mon cerveau.

La conclusion est explicite, puisqu'Edmond présente finalement Nephtalie comme une mère spirituelle: " c'est elle qui m'a donné le goût de la littérature. [...] Et certes, dans l'ouverture de mon esprit et dans la formation de mon talent futur, elle a fait cent fois plus que les illustres maîtres qu'on veut bien me donner ". Et l'influence de la tante semble avoir porté jusque sur le style de l'écrivain ("Dès ce temps, elle mettait en moi l'amour des vocables choisis, techniques, imagés , des vocables lumineux [...]"). On voit, dans tous les cas, le rôle matriciel joué par les demeures et leur décor.

103. On retrouve cet imaginaire du meuble de toilette qui mêle lumière, couleurs, matières précieuses : esthétique et sensualité (voir ensuite "la jouissance presque physique que j'avais à me laver les mains jusqu'aux coudes dans de la pâte d'amande "). 
Dernier passage du Journal qui croise le texte de La Maison d'un artiste, puisqu'il constitue un addenda qui aurait dû y être inséré dans une réédition projetée, la description du Grenier à la date du 14 décembre $1894^{104}$. Selon le modèle de La Maison, ce texte intègre en son milieu l'évocation de la bibliothèque, et celle-ci conduit aux livres des Goncourt eux-mêmes (les cinq volumes sous vitrine qui surmontent le rayonnage). En outre, le passage s'achève sur la description des livres-portraits, dont la série se clôt sur l'effigie d'Edmond par Carrière sur un exemplaire de Germinie Lacerteux, "dans lequel Carrière a merveilleusement exprimé la vie fiévreuse des yeux de l'auteur " : l'éloge du peintre est finalement un éloge du modèle et insiste sur sa capacité créatrice. Conclusion qui fait bien du Grenier un lieu profondément dédié à l'activité littéraire, non seulement dans son résultat, mais aussi dans sa dynamique ${ }^{105}$.

104. Journal, III, 1046.

105. A partir d'un croisement aussi manifeste entre ces deux textes, le Journal et la Maison d'un artiste, l'un intégrant ce qui devrait figurer dans l'autre, on peut plus largement $s$ 'interroger sur leur parenté. Il faut certes d'emblée évoquer les différences essentielles (au-delà dc leur différence évidente de proportion - 600 pages contre quelque 3000), puisque la Maison, au contraire du Journal, est centrée sur un individu et sur une année (1881). Mais des similitudes profondes existent. La Maison relève, comme le Journal, du genre des mémoires (" mémoires des choses au milieu desquelles s'est écoulée une existence d'homme "). La stratification historique existe bien dans les deux textes (le Journal, surtout centré sur l'histoire du présent, est aussi saturé de références à l'ancien; et la Maison, aux côtés du passé lointain de l'Ancien Régime, intègre aussi le passé proche, avec l'acquisition des objets, et l'histoire personnelle du collectionneur). La présence collective enfin est manifeste dans les deux textes : la Maison convoque une grande quantité de noms (artistes et acteurs du passé, historiens, collectionneurs et marchands du présent), les "autres" apparaissent bien, mais dans la dépendance du « je ». C'est pourquoi il faudrait mettre en parallèle la Maison et le Journal, et les considérer comme deux systèmes complémentaires occupant chacun une place essentielle dans l'œuvre des Goncourt dont ils constituent les deux socles : l'un est un réservoir d'observations (paroles entendues, choses vues), l'autre un réservoir d'objets (la Maison réunissant les pièces de la collection qui circulent dans les textes d'histoire et d'histoire de l'art). Ils assurent la cohérence formelle d'un vaste univers textuel, ils servent conjointement une stratégie de positionnement dans le champ littéraire. On pourrait en outre s'interroger sur leur interaction; pour ce qui est des descriptions de lieux, c'est bien parce qu'il y a eu la Maison que la teneur du Journal s'infléchit, et c'est parce qu'on avait eu des évocations de demeures dans le Journal que la Maison a été possible. 
La représentation des lieux, et particulièrement des intérieurs, est bien une problématique à la croisée des genres pratiqués par les Goncourt (roman, histoire, mémoires), à la croisée aussi des perspectives critiques (historique, sociologique, esthétique) qu'on peut adopter sur leur ouvre. Elle fait particulièrement émerger cette mise en abyme du fait littéraire, cette interrogation angoissée sur ses conditions de possibilité (d'où la nécessité de bâtir, de décrire le lieu idéal pour écrire), qui se sont imposées depuis la fin du XIX siècle parmi ces critères de la "modernité ", de la "littérarité ", aujourd'hui à redéfinir. Pour l'archéologie à venir de ces deux notions, on verrait qu'ici le neuf croise le plus ancien, et l'écriture la matérialité des choses...

Dominique PETY 